OPEN ACCESS

Edited by:

Guicai Li,

Nantong University, China

Reviewed by:

Da Huo,

Nanjing Medical University, China

Luzhong Zhang,

Nantong University, China

*Correspondence:

Changjiang Pan

panchangjiang@hyit.edu.cn

Wei Ye

weiye@hyit.edu.cn

Specialty section:

This article was submitted to Biomaterials,

a section of the journal

Frontiers in Bioengineering and Biotechnology

Received: 28 September 2021

Accepted: 21 October 2021

Published: 04 November 2021

Citation:

Liu S, Shen C, Qian C, Wang J, Wang Z, Tang X, Zhang Q, Pan C and Ye W (2021) A Rapid Dual-Responsive

Releasing Nano-Carrier by

Decomposing the Copolymer and

Reversing the Core Dissolution. Front. Bioeng. Biotechnol. 9:784838.

doi: $10.3389 /$ fbioe. 2021.784838

\section{A Rapid Dual-Responsive Releasing Nano-Carrier by Decomposing the Copolymer and Reversing the Core Dissolution}

\author{
Sen Liu ${ }^{1}$, Can Shen ${ }^{1}$, Cheng Qian ${ }^{1}$, Jianquan Wang ${ }^{2}$, Zhihao Wang ${ }^{1}$, Xuecong Tang ${ }^{1}$, \\ Qiuyang Zhang ${ }^{1}$, Changjiang Pan $^{1 *}$ and Wei Ye ${ }^{1 *}$
}

${ }^{1}$ Jiangsu Provincial Engineering Research Center for Biomedical Materials and Advanced Medical Devices, Faculty of Mechanical and Material Engineering, Huaiyin Institute of Technology, Huaian, China, ${ }^{2}$ College of Engineering and Applied Sciences, Institute of Materials Engineering, Collaborative Innovation Center of Chemistry for Life Sciences, Nanjing University, Nanjing, China

The accumulation of nanotechnology-based drugs has been realized in various ways. However, the concentration of drugs encapsulated by nanomaterials is not equal to the concentration of effective drugs; often, the drugs become effective only when they are released from the nanomaterials as free drugs. This means only when the drugs are rapidly released after the accumulated drug-encapsulating nanomaterials can they truly achieve the purpose of increasing the concentration of drugs in the tumor. Therefore, we herein report a dual-response nano-carrier of glutathione and acid to achieve the rapid release of encapsulated drug and increase the effective drug concentration in the tumor. The nanocarrier was constructed using a dual-responsive amphiphilic copolymer, composed of polyethylene glycol and hydrophobic acetylated dextran and connected by a disulfide bond. In the tumor microenvironment, disulfide bonds could be biodegraded by glutathione that is overexpressed in the tumor, exposing the core of nano-carrier composed of acetylated dextran. Then the acidic environment would induce the deacetylation of acetylated dextran into water-soluble dextran. In this way, the nanocarrier will degrade quickly, realizing the purpose of rapid drug release. The results showed that the drug release rate of dual-responsive nano-carrier was much higher than that of glutathione or acid-responsive nano-carrier alone. Furthermore, both in vitro and in vivo experiments confirmed that dual-responsive nano-carrier possessed more efficient antitumor effects. Therefore, we believe that dual-responsive nano-carriers have better clinical application prospects.

Keywords: dual-responsive, nano-carrier, dissolution reversing, rapid drug release, tumor chemotherapy

\section{INTRODUCTION}

Nano-carriers have played significant roles in improving the therapeutic effects of tumor chemotherapy in the past decades (Petros and Desimone, 2010; Shi et al., 2016). Therefore, currently, it is one of the most interesting research directions to explore more effective drug carriers to overcome tumors (Sun et al., 2017; Yao et al., 2020; Zhang et al., 2021). The rapid release of nano-carriers at the lesion site is an important property of ideal nano-carriers (Hou et al., 2019). Drugs exhibit their therapeutic activity only after being liberated from their carrier (Luck and Mason, 


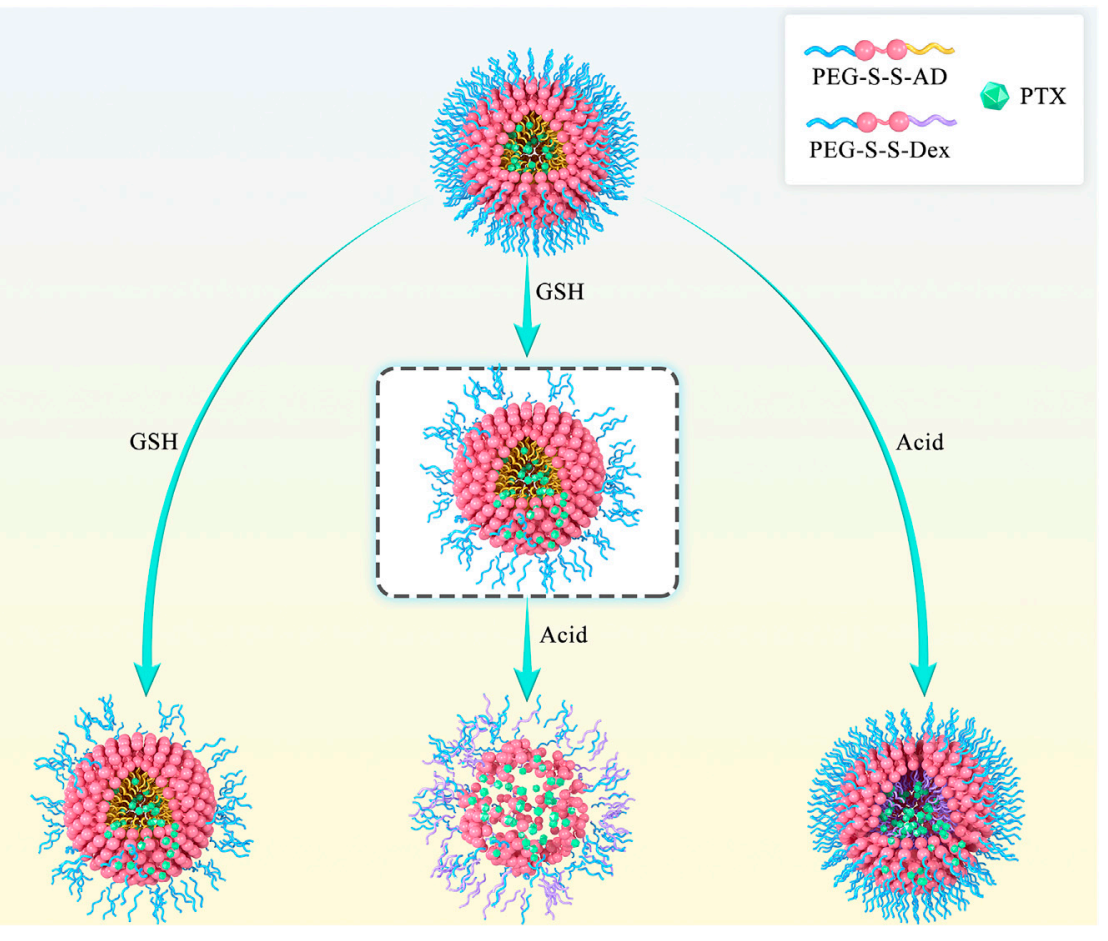

FIGURE 1 | Scheme of rapid cleavage of dual-responsive nano-medicine (PTX@PSA NPs) in the presence of glutathione and acid. Compared to the shedding of the outer PEG layer induced by the breaking of disulfide bond in the presence of glutathione alone and the internal expansion induced by the change of hydrophobic AD to hydrophilic Dex in the presence of acid alone, dual-responsive PTX@PSA NPs possess a faster drug release rate.

2013), and cancer cells have multiple mechanisms of multi-drug resistance to protect them from drugs, such as drug efflux (Ye et al., 2017; Liu et al., 2019), cell-intrinsic drug metabolism (Colombo et al., 1999), or detoxification (Wegiel et al., 2013). Thus, the drug release rate should be rapid enough to achieve a sufficiently high concentration in tumor cells, allowing the drugs to reach their targets and kill cancer cells.

Usually, the drugs are released from the carrier to become free drugs through the drug diffusion (Colombo et al., 1999) or carrier disintegration (Colombo et al., 1999; Yu et al., 2015a; Liu et al., 2019; Zhang et al., 2019; Cao et al., 2021) mechanisms. However, the rate of drug diffusion is relatively slow, usually with a half-life of several hours or days after release (Sun et al., 2012). Therefore, the carrier can be designed with the characteristics of rapid response dissociation so that they can quickly become free drugs at the lesion site to play their role in tumor inhibition.

Herein, we report a dual-responsive amphiphilic copolymer (PEG-SS-AD), composed of polyethylene glycol (PEG) and acetylated dextran (AD) linked by a disulfide bond (-S-S-). The rapid dual-responsive releasing nano-carrier (PSA NPs) was formed by the self-assembly of PEG-SS-AD. It is wellestablished that tumor cells overexpress glutathione (GSH) (Yan et al., 1991; Hou et al., 2019), which could act on -S-Sthrough biodegrading (Lappi and Ruddock, 2011; Hou et al., 2019). Therefore, the nano-carrier would degrade at the tumor site, and the water-soluble outer shell would be separated and dissolved in the -S-S- biodegrading process. The hydrophobic part was functionalized with acid-induced solubility reversible ability to accelerate the disintegration of the exposed hydrophobic core. Hydrophobic acetylated dextran (Dex) can be converted into hydrophilic Dex in the tumor's acidic microenvironment (Dai et al., 2019). Hence, the dual-responsive nano-carrier is capable of rapid cleavage in the tumor (Figure 1). Once loaded with chemotherapeutic drugs, these nano-carriers can quickly release the coated drugs at the tumor site, increasing the concentration of the drug at the tumor site to enhance the effectiveness of chemotherapy.

\section{EXPERIMENTAL SECTION}

\section{Materials}

Methoxy-terminated polyethylene glycol (mPEG, $\mathrm{Mw} \approx 2 \mathrm{KDa}$ ), carboxylic acid functionalized methoxyl polyethylene glycol (mPEG-COOH, Mw $\approx 2 \mathrm{KDa}$ ) were purchased from Ruixi Biological Technology Co., Ltd,. (Xi'an, China). N,N'carbonyldiimidazole (CDI), 2,2-dithiodiethanol (DIT), succinic anhydride, dextran ( $\mathrm{Mw} \approx 2 \mathrm{KDa}), 1$-(3-Dimethylaminopropyl)3-ethylcarbodiimide hydrochloride (EDC), N-Hydroxy succinimide (NHS), 2-methoxy propylene, pyridine p-toluene sulfonate, propionic anhydride, and dimethylaminopyridine (DMAP) were purchased from Energy Chemical (Shanghai, China). Water used in all the experiments was deionized (DI) water depurated by a Millipore ultrapure water system (Billerica, 
MA, United States) with a resistivity of $18.2 \mathrm{M} \Omega \mathrm{cm}$. Highglucose DMEM containing $1 \%$ penicillin/streptomycin, phosphate-buffered saline (PBS), and trypsin were obtained from KeyGen BioTech Co., Ltd,. (Jiangsu, China). Fetal bovine serum (FBS) was purchased from Absin Bioscience Inc (Shanghai, China).

\section{Synthesis of PEG-SS-AD (PSA)}

Briefly, $4.0 \mathrm{~g}$ of mPEG and $0.162 \mathrm{~g}$ of CDI were dissolved in $50 \mathrm{ml}$ of N,N-dimethyl formamide (DMF) and stirred for $24 \mathrm{~h}$ at room temperature. Then, $0.26 \mathrm{~g}$ of DIT was added and reacted at room temperature for another $12 \mathrm{~h}$. The byproduct was then dialyzed and lyophilized to obtain disulfide bond-functionalized PEG (mPEG-SS, polymer 2).

Then, $2.2 \mathrm{~g}$ of mPEG-SS, dissolved in $50 \mathrm{ml}$ DMF, was dissolved in $20 \mathrm{ml}$ of DMF. Next, excess succinic anhydride was added and stirred at room temperature for $24 \mathrm{~h}$. The byproduct was then dialyzed and lyophilized to obtain carboxyl-functionalized mPEG-SS (mPEG-SS-COOH, polymer 3 ).

In the next stage, $1.0 \mathrm{~g}$ of mPEG-SS-COOH and $1.0 \mathrm{~g}$ of Dex were dissolved in $20 \mathrm{ml}$ of DMF, and $0.1 \mathrm{~g}$ of EDC and $0.06 \mathrm{~g}$ of NHS were added as catalysts. After $24 \mathrm{~h}$ of reaction, the byproduct was dialyzed and lyophilized to obtain PEG-SS-Dex (polymer 4).

Subsequently, $0.1 \mathrm{~g}$ of PEG-SS-Dex, $10 \mathrm{ml}$ of 2-methoxy propylene, and $50 \mathrm{mg}$ of pyridine p-toluene sulfonate were dissolved in $20 \mathrm{ml}$ of dimethyl sulfoxide (DMSO). Then, the reactions were carried out at room temperature for $3 \mathrm{~h}$. The byproduct was dialyzed and lyophilized to obtain PEG-SS-AD (polymer 5).

${ }^{1} \mathrm{H}-\mathrm{NMR}$ of the polymers was performed on a Bruker $400 \mathrm{M}$ (JNM-ECZS) with the $\mathrm{CDCl}_{3}$ solvent.

\section{Synthesis of PEG-propionic anhydride-modified dextran (PPD)}

To synthesize PDP as a non-responsive control group, $1.2 \mathrm{~g}$ of mPEG-COOH, $1.0 \mathrm{~g}$ of Dex, and $97.5 \mathrm{mg}$ of CDI were dissolved in $20 \mathrm{ml}$ of DMSO, and the reactions were allowed to continue under stirring at $80^{\circ} \mathrm{C}$ overnight. Then, $5 \mathrm{ml}$ of propionic anhydride, $100 \mathrm{mg}$ of DMAP, and $1 \mathrm{ml}$ of triethylamine were mixed and stirred at room temperature for $8 \mathrm{~h}$. PPD was then obtained by dialysis and lyophilization.

\section{Preparation of Nano-Carriers (PSA NPs) and Nano-Medicine (PTX@PSA NPs)}

PSA NPs were prepared by the self-assembly of PEG-SS-AD in the selective solvent of dichloromethane (DCM) in DI water, as reported previously (Hu et al., 2007; Yu et al., 2015b). Briefly, $10 \mathrm{mg}$ of PEG-SS-AD was dissolved in $1 \mathrm{ml}$ of DCM, and the mixture was slowly added to $10 \mathrm{ml}$ of DI water. The mixture was then emulsified by ultra-sonication for $10 \mathrm{~min}$. Finally, the emulsion was evaporated to remove DCM, followed by filtration and lyophilization. PTX@PSA NPs were obtained by adding $10 \mathrm{mg}$ of PEG-SS-AD and $2 \mathrm{mg}$ of PTX in $1 \mathrm{ml}$ of DCM, and then the steps above were followed. Non-responsive PTX@ PPD NPs were obtained by adding $10 \mathrm{mg}$ of PEG-PD and $2 \mathrm{mg}$ of PTX to $1 \mathrm{ml}$ of DCM. Then the steps above were followed.

\section{Characterization of PSA NPs}

To this end, $10 \mathrm{mg}$ of PSA NPs was dissolved in $10 \mathrm{ml}$ of alkalescent $(\mathrm{pH} \approx 7.3)$ phosphate-buffered saline (PBS) solution, $0.2 \mu \mathrm{mol} / \mathrm{L}$ GSH alkalescent $(\mathrm{pH} \approx 7.3)$ solution, acidic $(\mathrm{pH} \approx 6.0)$ PBS solution, and $0.2 \mu \mathrm{mol} / \mathrm{L}$ GSH $(\mathrm{pH}$ $\approx 6.0$ ) PBS solution, respectively, and incubated for $2 \mathrm{~h}$. In addition, transmission electron microscopic (TEM, JEM$2100 \mathrm{~F}$ ) images were obtained to analyze changes in the morphology of PSA NPs under different conditions.

\section{Drug Loading Content and Encapsulation Efficiency}

Standard solutions of PTX at concentrations of $0.001,0.005,0.01$, $0.05,0.1,0.5$, and $1 \mathrm{mg} / \mathrm{ml}$ were prepared to measure their ultraviolet absorption spectrum and evaluate the relationship between concentration and absorbance at $273 \mathrm{~nm}$.

Then $10 \mathrm{mg}$ of PTX@PSA NPs (W dissolved in $4 \mathrm{ml}$ of acetonitrile and $\mathrm{PBS}(\mathrm{pH}=7.4)(\mathrm{V} / \mathrm{V}=2: 3)$ mixed solvent and the supernatant was centrifuged. Then the concentration of PTX ( $\left.\mathrm{C}_{\mathrm{PTX}}\right)$ was measured by absorption photometry. The PTX loading content and encapsulation efficiency were calculated by (Eqs. 1, 2)

$$
\begin{gathered}
\text { PTX loading content }=\frac{C_{P T X} \times 4 m L}{W_{P T X @ P S A N P s}} \times 100 \% \\
\text { PTX encapsulation efficiency }=\frac{C_{P T X} \times 4 m L}{\text { total amount of PTX }} \times 100 \%
\end{gathered}
$$

where the total amount of PTX $=\mathrm{W}_{\mathrm{PTX} @ \mathrm{PSA} \mathrm{NPs}} / 6=1.67 \mathrm{mg}$, according to the feeding ratio.

\section{In Vitro Controlled Release Ability}

First, $5 \mathrm{ml}$ of PTX@PSA NPs aqueous solutions $(2 \mathrm{mg} / \mathrm{ml})$ were sealed into dialysis tubes (MWCO $2 \mathrm{kDa}$ ) for four copies, and then immersed in $45 \mathrm{ml}$ of alkalescent $(\mathrm{pH} \approx 7.3$ ) phosphatebuffered saline (PBS) solution, $0.2 \mu \mathrm{mol} / \mathrm{L}$ GSH alkalescent $(\mathrm{pH}$ $\approx 7.3$ ) PBS solution, acidic ( $\mathrm{pH} \approx 6.0$ ) PBS solution, and $0.2 \mu \mathrm{mol} /$ L GSH acidic ( $\mathrm{pH} \approx 6.0$ ) PBS solution, respectively. The drug release experiment was performed at $37^{\circ} \mathrm{C}$, and three $1-\mathrm{ml}$ solutions were taken from each group at $10 \mathrm{~min}$ and $0.5,1,3$, $5,8,12$, and $24 \mathrm{~h}$, respectively. The concentration of PTX was measured by ultraviolet absorption spectrum, and the cumulative released PTX was calculated.

\section{In vitro cytotoxicity assay and anti-tumor effect}

The cytotoxicity of $4 \mathrm{~T} 1$ cells was tested using an MTT (3-(4,5dimethylthiazol-2-yl)-2,5-diphenyltetrazolium bromide) assay. The cells were inoculated in 96-well plates, and when the cells covered approximately $80 \%$ of the total area, the medium was 


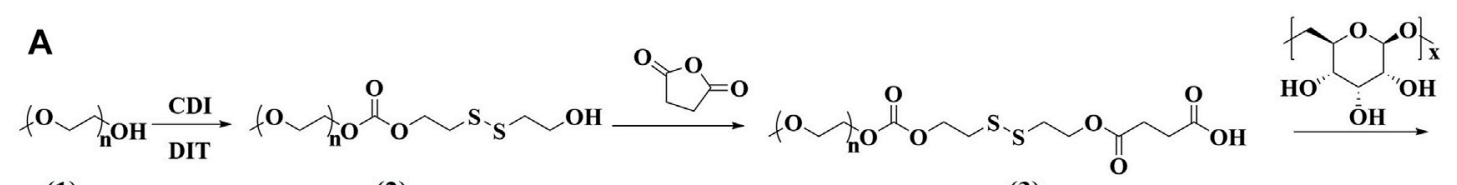

(1)

(2)

(3)

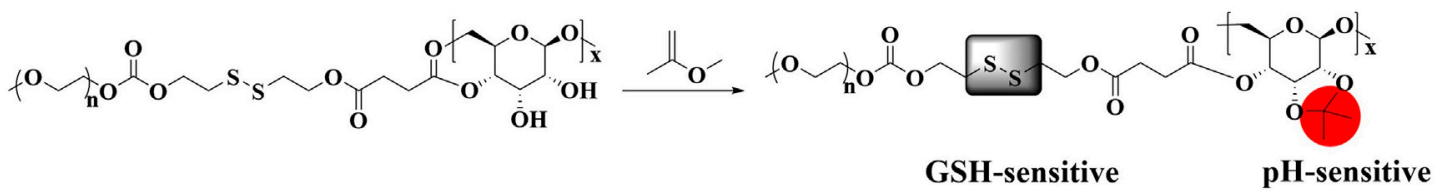

(4)

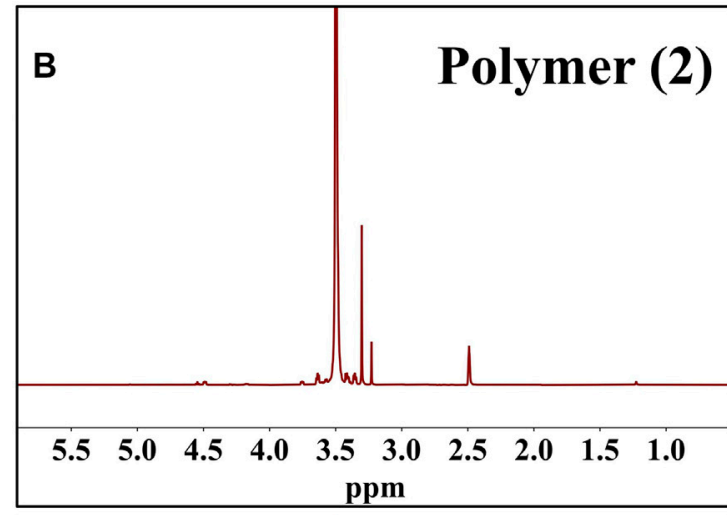

(5)
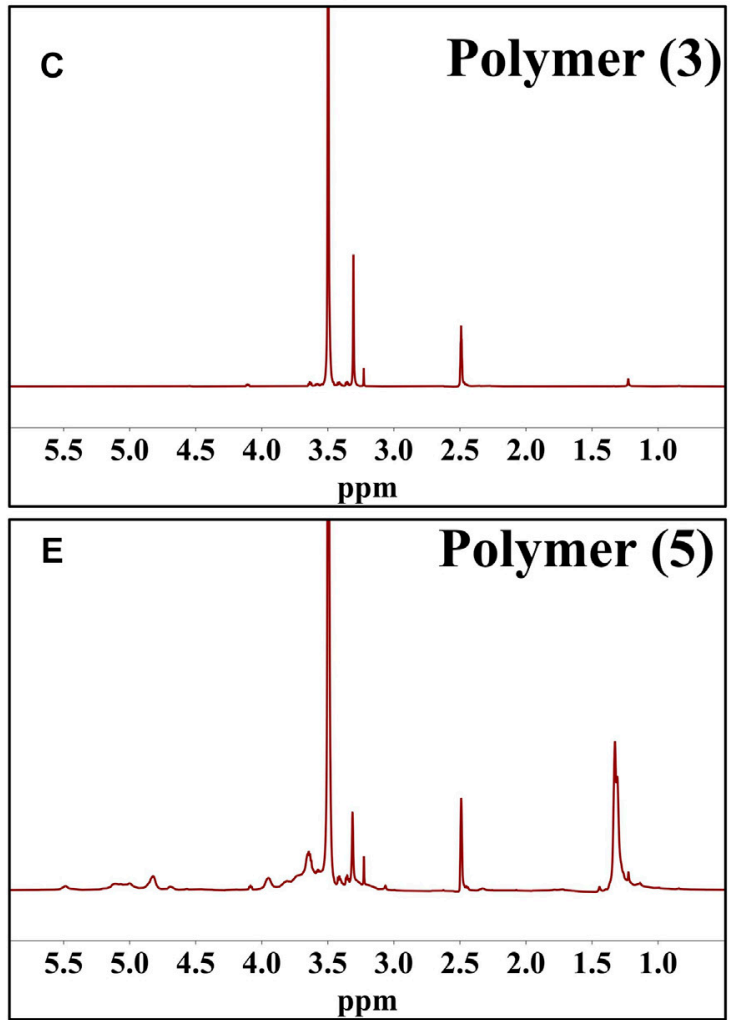

FIGURE 2 | (A) Synthetic route of dual-responsive amphiphilic polymer; 1H NMR spectrum of polymers 2 to 5 (B-E).

removed, and $100 \mu \mathrm{l}$ of PSA NPs medium solution was added at concentrations of $0,10,10^{2}, 10^{3}, 10^{4}, 10^{5}$, and $10^{6} \mathrm{ng} / \mathrm{ml}$. After incubation for $24 \mathrm{~h}$, the medium was removed, and the cells were washed three times with saline solution. Then $20 \mu \mathrm{l}$ of MTT solution ( $2.5 \mathrm{mg} \mathrm{ml}-{ }^{1}$ in saline solution) and $80 \mu \mathrm{l}$ of culture medium were added per well, and the cells were incubated for another $4 \mathrm{~h}$. Subsequently, the medium was aspirated, and $200 \mu \mathrm{l}$ of DMSO solution was added to each well. After $15 \mathrm{~min}$, the absorbance was measured at $490 \mathrm{~nm}$ using an iMark Enzyme mark instrument (Biotek Eon ${ }^{\mathrm{TM}}$ ). The cell viability was calculated according to a previously reported approach.

The anti-tumor effects of PTX@PSA NPs and PTX@PPD NPs were tested using MTT assay by following the above method.

\section{In vivo Anti-Tumor Effects}

A tumor-bearing mouse model was constructed by injecting 4T1 into the back of the mouse. When the tumor grew to approximately $100 \mathrm{~mm}^{3}$, they were divided into three groups $(n=10)$. Each mouse was intravenously injected with saline solution $(100 \mu \mathrm{l})$ (group 1), PTX@PPD NPs $(100 \mu \mathrm{l}, 0.5 \mathrm{mg} / \mathrm{ml})$ (group 2), and PTX@PSA NPs (100 $\mu \mathrm{l}, 0.5 \mathrm{mg} / \mathrm{ml}$ ) (group 1). The treatment was performed twice a week for two weeks. Then the length ( $\mathrm{L}$, longest diameter) and width (W, shortest diameter) of the tumor were measured with vernier calipers every 3 days from the beginning of treatment, and the tumor volume was calculated using this Eq. (3):

$$
V=\left(L \times W^{2}\right) / 2
$$



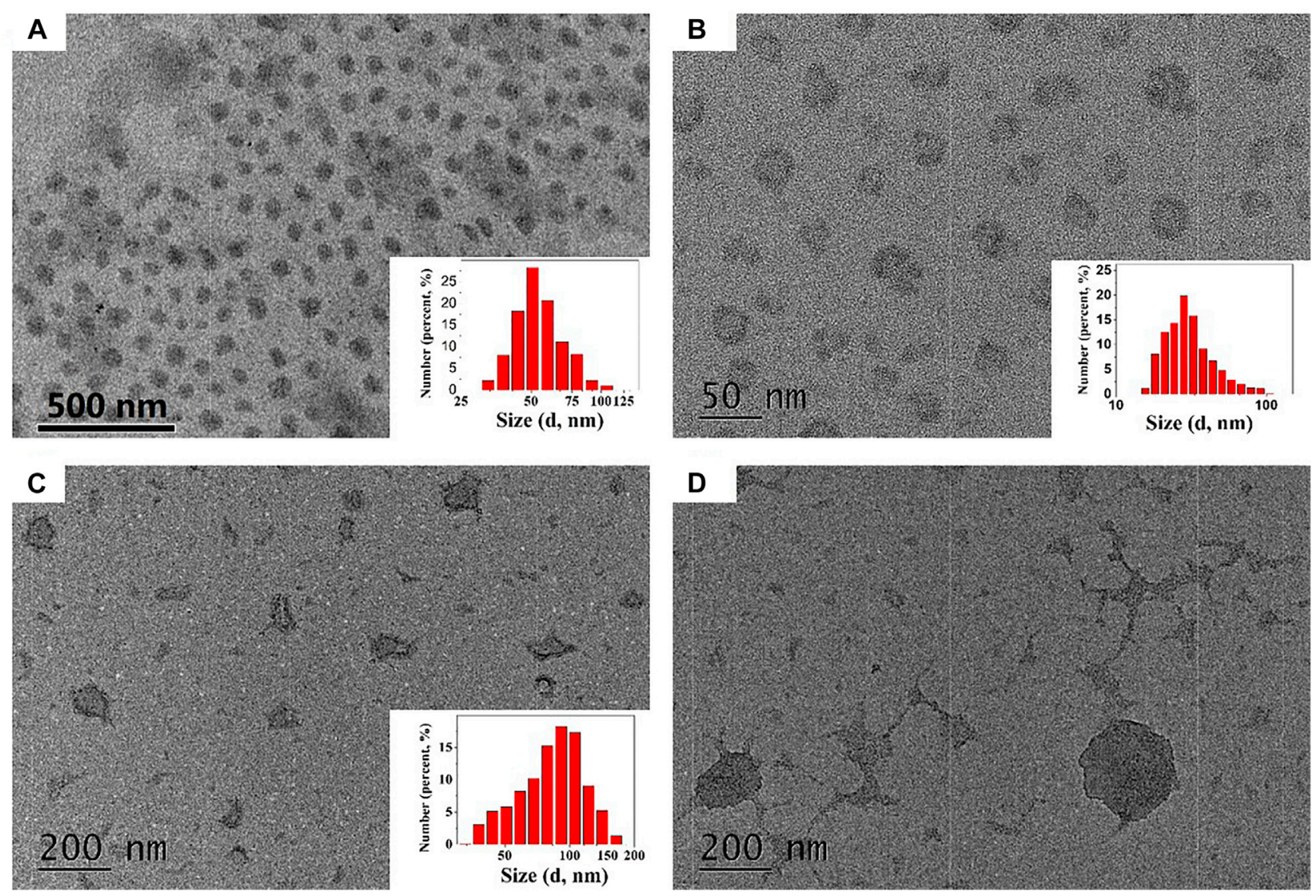

FIGURE 3 | (A) The TEM images of PEG-SS-AD NPs (PSA NPS). (B) The TEM images of PEG-SS-AD NPs incubated in $0.2 \mu$ mol/L GSH alkalescent (pH $\approx 7.3$ ) phosphate-buffered saline (PBS) solution. (C) The TEM images of PEG-SS-AD NPs incubated in acidic (pH $\approx 6.0$ ) PBS solution. (D) The TEM images of PEG-SS-AD NPS incubated in $0.2 \mu \mathrm{mol} / \mathrm{L}$ GSH acidic $(\mathrm{pH} \approx 6.0)$ PBS solution.

Three of the tumors in each group were harvested, and their optical pictures were obtained. The survival of the mice was determined every 3 days.

Three tumor-bearing mice were randomly selected and treated according to the above procedures. Tumors were harvested, isolated, immobilized, embedded into paraffin, cut into sections, stained with $\mathrm{H} \& \mathrm{E}$, and observed under a microscope on the 14th day.

\section{Pathological Analysis}

The tumor-bearing mice were intravenously injected with PSA NPs and PTX@PSA NPs, and then sacrificed on the 14th day. The main organs (heart, liver, spleen, lungs, kidneys) were harvested, isolated, fixed, embedded into paraffin, cut into sections, stained by $\mathrm{H} \& \mathrm{E}$, and observed under a microscope.

\section{RESULTS AND DISCUSSION}

\section{Synthesis and Characterization of Dual-Responsive Amphiphilic Copolymers}

Figure 2A shows the synthesis pathway of $\mathrm{GSH}$ and $\mathrm{pH}$ dualresponsive PEG-SS-AD (Polymer 5). The GSH response was achieved by incorporating disulfide bonds between polyethylene glycol $(\mathrm{PEG}, \mathrm{Mw} \approx 2 \mathrm{KDa})$ and $\mathrm{Dex}(\mathrm{Mw} \approx 2 \mathrm{KDa})$ copolymer, while the $\mathrm{pH}$ response was achieved by acetylated dextran, which could be converted into hydrophilic dextran in the tumor's acidic microenvironment. The products of polymers $2-5$ were confirmed at each step by ${ }^{1} \mathrm{H}$ NMR (Figures $2 \mathbf{B}-\mathbf{E}$ ). The absorption peak at $3.50 \mathrm{ppm}$ represents the presence of PEG. The absorption peak of Dex at 4.48-4.92 ppm, which could be attributed to $\mathrm{H}$ atoms in $-\mathrm{OH}$ in each monomer of Dex, was detected in polymer 4, indicating that Dex was attached to PEG. In addition, after the acetylated dextran, the absorption peak of methyl $\left(-\mathrm{CH}_{3}\right.$, the red part of polymer five in Figure 2A) appeared at $1.33 \mathrm{ppm}$, and the absorption peaks of $-\mathrm{OH}$ in Dex weakened, indicating the successful preparation of $\mathrm{AD}$. These findings showed that the GSH and $\mathrm{pH}$ dual-responsive PEG-SS-AD were successfully synthesized according to the synthetic route.

\section{Characterization of Dual-Responsive Nano-Carriers}

Then the nano-carriers were formed by the self-assembly of PEG-SS$\mathrm{AD}$ in a selective solvent (PSA NPs), using the solvent evaporation method as we reported previously (Hu et al., 2007; Yu et al., 2015b). As shown in Figure 3A, PSA NPs have good water dispersion with 

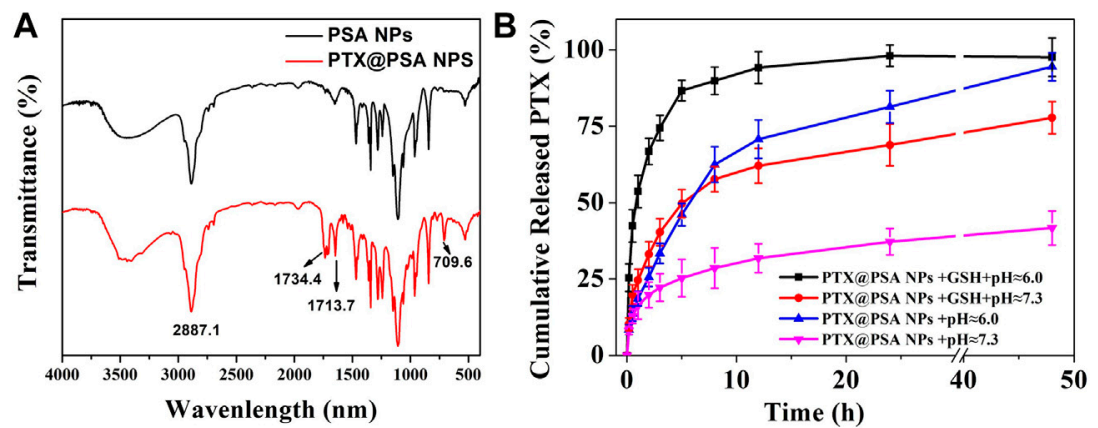

FIGURE 4 | (A) FT-IR spectra of the nano-carriers (PSA NPs) and PTX encapsulation nano-medicine (PTX@PSA NPs). (B) In vitro release of CO from PTX@PSA NPs under stimulation without GSH and acid (PTX@PSA NPs + pH $\approx 7.3$ ), acid (PTX@PSA NPs + pH $\approx 6.0)$, GSH (PTX@PSA NPs + GSH + pH $\approx 7.3)$, GSH and acid (PTX@PSA NPs + GSH + pH $\approx 6.0$ ).

TABLE 1 | The PTX loading content and encapsulation efficiency of PTX@ PSA NPS.

\begin{tabular}{lrcc}
$\begin{array}{l}\text { Weight of the } \\
\text { PTX@PSA }\end{array}$ & $\begin{array}{c}\text { Concentration } \\
\text { of PTX }(\boldsymbol{\mu g} / \mathbf{m l})\end{array}$ & $\begin{array}{c}\text { PTX loading } \\
\text { content }(\mathbf{w t} \%)\end{array}$ & $\begin{array}{c}\text { PTX encapsulation } \\
\text { efficiency } \\
\text { (wt } \%)\end{array}$ \\
\hline 10 & 292.5 & 11.7 & 70.2
\end{tabular}

relatively uniform size, i.e., approximately $40 \mathrm{~nm}$. The mean hydrodynamic diameter of these PSA NPs was measured by dynamic light scattering (DLS), which was $55 \mathrm{~nm}$ with a polydispersity index (PdI) of 0.068 (inset). Then, PSA NPs were incubated in different solutions for $30 \mathrm{~min}$, and their morphologies were observed under TEM, and mean hydrodynamic diameter was measured by DLS (Figure 3B-D). After incubating in $0.2 \mu \mathrm{mol} / \mathrm{L}$ GSH alkalescence $(\mathrm{pH} \approx 7.3)$ phosphate-buffered saline (PBS) solution, the morphology of the PSA NPs was no longer regular, and some PSA NPs were worn out, and others were shrunk in size, with a mean hydrodynamic diameter of $41 \mathrm{~nm}$ and a higher PdI of 0.238 (Figure 3B). This might be due to the cleavage of -S-S- in PEG$\mathrm{SS}-\mathrm{AD}$ by GSH and the dissolution of the PEG shell. In addition, after incubating in acidic ( $\mathrm{pH} \approx 6.0$ ) PBS solution without $\mathrm{GSH}$, significant changes were observed in the morphology of PSA NPs, and more organic debris appeared, with a mean hydrodynamic diameter of $82.7 \mathrm{~nm}$ and a much higher PdI of 0.452 (Figure 3C). This might be attributed to the transition from hydrophobic AD to hydrophilic Dex in the acidic environment and the nano-carriers dissolved by the solvent. However, after incubating in $0.2 \mu \mathrm{mol} / \mathrm{L}$ GSH acidic $(\mathrm{pH} \approx 6.0)$ PBS solution, there were no nano-carriers anymore, and all the nano-carriers degraded to organic fragments (Figure 3D). These findings indicated that PSA NPs possess the dual-responsive characteristics of GSH and acid and could disintegrate rapidly under the dual action of GSH and acid compared to GSH or acid alone. Encapsulation and controlled release properties of PTX@PSA NPs.

As PSA NPs possess dual-responsive characteristics of GSH and acid, PSA NPs encapsulating paclitaxel (PTX) (PTX@PSA NPs) were prepared, and the encapsulation and controlled release properties of PTX@PSA NPs were evaluated.
To prove the successful encapsulation of PTX by PSA NPs, the as-prepared PTX@PSA NPs were centrifuged and re-dispersed three times to fully remove the free PTX.FT-IR of PTX@PSA NPs and PSA NPs were then carried out, as shown in Figure 4A. The appearance of vibration peaks of the carbonyl group (1734.4, $\left.1713.7 \mathrm{~cm}^{-1}\right)$ and aromatic ring peak $\left(709.6 \mathrm{~cm}^{-1}\right)$ of PTX indicates that PTX was successfully encapsulated in PTX@PSA NPs. Through further testing, the PTX loading content and encapsulation efficiency of PTX@PSA NPs were calculated at 11.7 and 70.6\%, according to Eqs. 1, 2 (Table 1).

The cumulative in vitro release of PTX from PTX@PSA NPs was then measured under different conditions (Figure 4B). The release rate of PTX from PTX@PSA NPs was relatively slow in the absence of GSH and acid ( $\mathrm{pH} \approx 7.3$ ); $19.8 \pm 4.2 \%$ of the total amount was released in the first $2 \mathrm{~h}$, most of which were probably the PTX adhering to the surface of PTX@PSA NPs, and only $41.7 \pm 5.6 \%$ of the total amount was released at $48 \mathrm{~h}$. Under the stimulation of GSH $(\mathrm{GSH}+\mathrm{pH} \approx 7.3)$ or acid $(\mathrm{pH} \approx 6.0)$ alone, the PTX release rate increased, with $25.6 \pm 3.1 \%$ and $33.1 \pm 4.1 \%$ being released within $2 \mathrm{~h}$, and $94.5 \pm 4.6 \%$ and $77.8 \pm 5.3 \%$ being released at $48 \mathrm{~h}$, respectively. In contrast, under the combined action of GSH and acid $(\mathrm{GSH}+\mathrm{pH} \approx 6.0)$, the release rate of PTX increased significantly; $66.8 \pm 4.3 \%$ of the total amount was released within $2 \mathrm{~h}$, and almost all the drug was released after $12 \mathrm{~h}(94.2 \pm 5.2 \%)$. The results were consistent with the morphologies of the nano-carriers under different conditions in Figure 3. The nano-carriers remained relatively stable under the weak alkaline condition without GSH, and the drug release rate was slow. Part of the nano-carriers degraded under the stimulation of GSH or acid, resulting in an increased PTX release rate. Under the dual stimulation of GSH and acid, the nanocarriers degraded rapidly, and the corresponding controlled release rate of drugs was very fast, releasing the coated PTX in a short time.

It is well-established that the tumor microenvironment is acidic, and overexpressed GSH, the dual-responsive nanocarriers designed here can rapidly degrade and release the encapsulated drugs into the tumor.

\section{In vitro Analysis}

Although PEG and dextran have good biocompatibility, (Mura et al., 2013; Zhang et al., 2014) the safety of nano-carriers should 

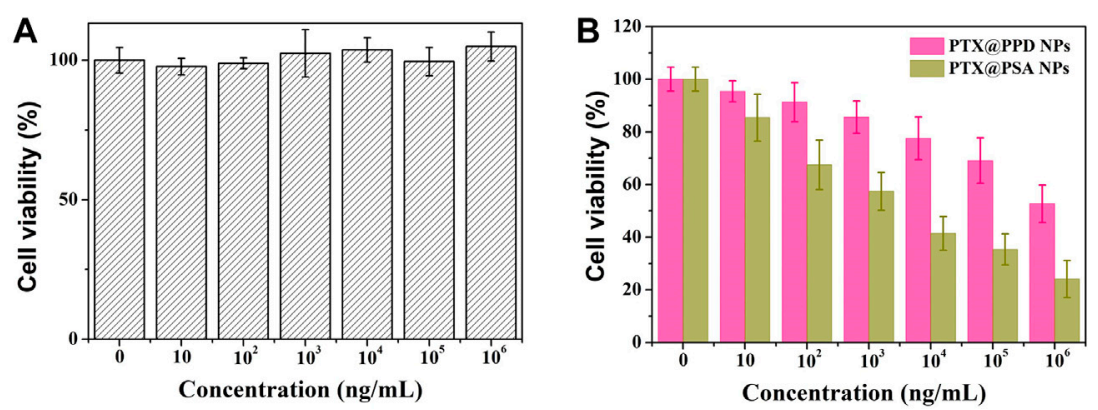

FIGURE 5 | (A) Cytotoxicity profiles of 4T1 cells. (B) In vitro cell proliferation-inhibiting efficiency of PTX@PPD NPs and PTX@PSA NPs on 4T1 cells.
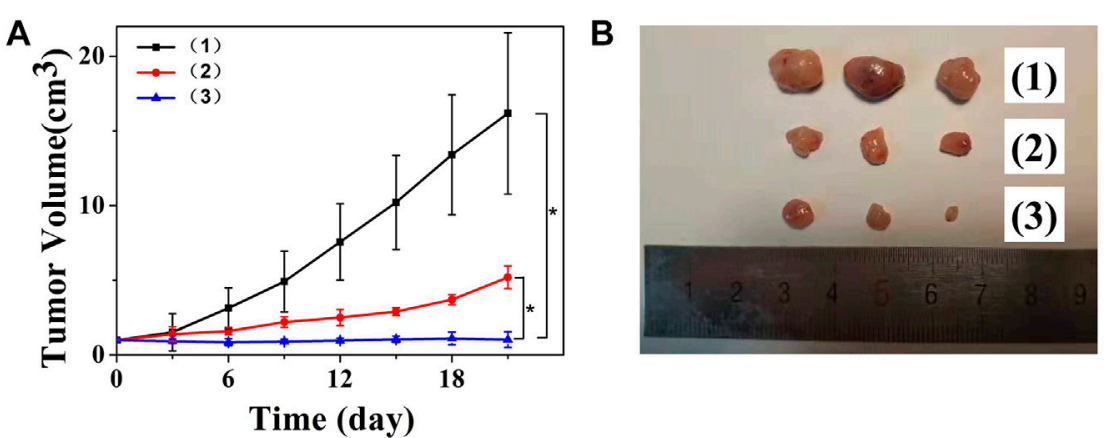

Time (day)

D

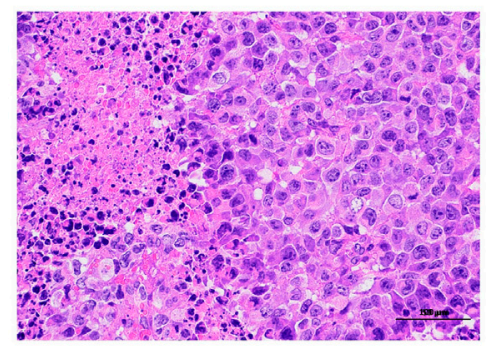

Group 2

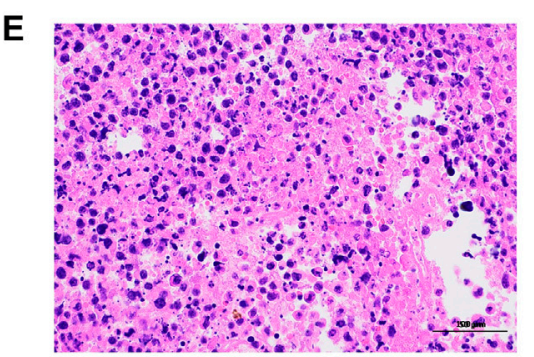

Group 3

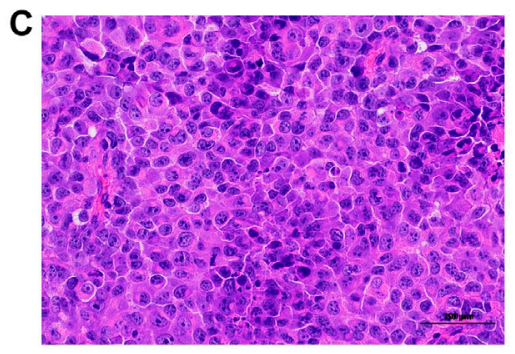

Group 1

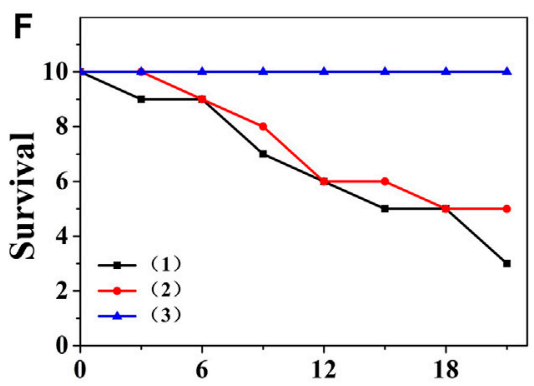

Time (day)

FIGURE 6 | (A) Changes in tumor volumes of mice receiving different treatments. ${ }^{*} p<0.01$. (B) Tumor images after treatment and observation period. (C) Pathological section of tumor tissue from group 1 via H\&E staining. (D) Pathological section of tumor tissue from group 2 via $\mathrm{H} \& E$ staining. (E) Pathological section of tumor tissue from group 3 via H\&E staining. Scale bars: $100 \mu \mathrm{m}$. (F) Survival curves of mice in each group ( $n=10$ each group). Each mouse was intravenously injected

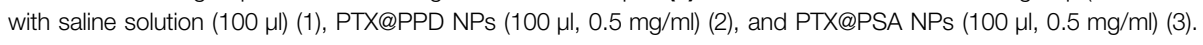

be evaluated. The cytotoxicity of the PSA NPs was evaluated at different concentrations through the MTT assay using $4 \mathrm{~T} 1$ cells. As shown in Figure 5A, PSA NPs showed no cytotoxicity. After co-culturing PSA NPs with $4 \mathrm{~T} 1$ cells, cell proliferation was almost not inhibited under various concentrations of PSA NPs solution, and the cell's survival rate was $104.9 \pm 5.2 \%$ at a relatively high concentration of $1 \mathrm{mg} / \mathrm{ml}$.

Then, propionic anhydride-modified dextran (PD), with no acid response characteristics, was successfully synthesized and directly connected to PEG without -S-S- to form a non-responsive amphiphilic copolymer (PEG-PD). Then non-responsive nanomedicine was prepared by PEG-PD encapsulating PTX (PTX@
PPD NPs). Then the in vitro cell proliferation-inhibiting efficiency of PTX@PPD NPs and PTX@PSA NPs was evaluated. As shown in Figure 5B, both PTX@PPD NPs and PTX@PSA NPs showed cytotoxicity, but PTX@PSA NPs exhibited higher inhibitory efficiency on the proliferation of tumor cells. This might be because PTX@PSA NPs has a faster controlled release rate, forming a higher concentration of PTX, with a better tumor cell inhibition efficiency.

\section{In vivo Anti-Tumor Effects}

Due to the EPR effect of solid tumors, nanoparticles measuring approximately $100 \mathrm{~nm}$ would accumulate in tumors (Lammers et al., 2012; Maeda et al., 2013; Wu, 2021). As the rapid, controlled 


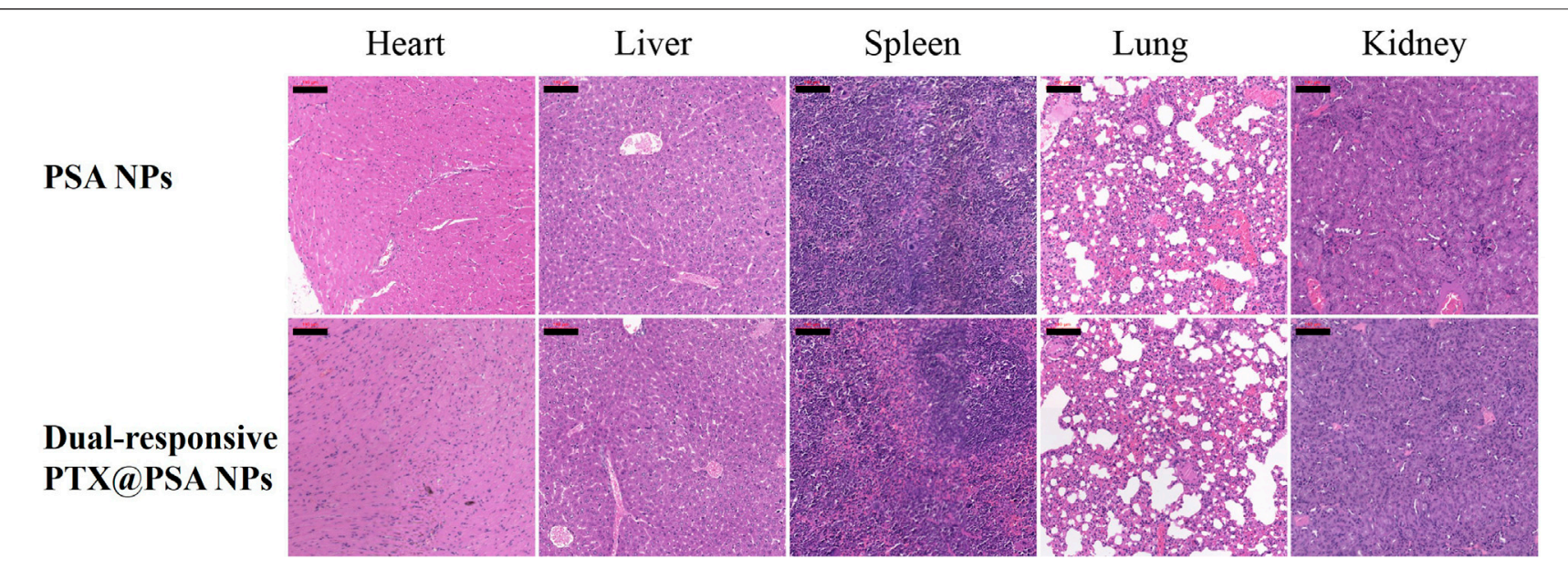

FIGURE 7 | The cytotoxic effect caused by PSA NPs and PTX@PSA NPs in major organs via H\&E staining (heart, liver, spleen, lungs, and kidneys). Scale bars: $100 \mu \mathrm{m}$.

release of PTX, PTX@PSA NPs showed could rapidly increase drug concentration and enhancing tumor cell inhibition in vitro. Thus, we speculated that PTX@PSA NPs would also show a significant anti-tumor effect in vivo. When the tumor size reached $\sim 100 \mathrm{~mm}^{3}$, tumor-bearing mice were equally divided into three groups $(n=10)$ and injected with normal saline solution $(100 \mu \mathrm{l})$, PTX@PPD NPs, and PTX@PSA NPs, twice a week for 2 weeks.

The tumor volume was measured and calculated every 3 days, and the statistical results are presented in Figure 6A. The tumor volume in mice injected with saline solution increased rapidly. The tumor volume in mice injected with non-responsive PTX@ PPD NPs was suppressed but still grew to $520 \pm 76 \mathrm{~mm}^{3}$ on day 21. Dual-responsive PTX@PSA NPs showed promising tumor inhibition, and the tumor volume did not increase; it even decreased during the statistical period within 21 days. On day 21 , optical camera images of three randomly selected tumors, presented in Figure 6B, showed the therapeutic effect. To further illustrate the effect of treatment on the tumor, three tumorbearing mice were treated according to the above groups, and the tumoral sections of mice were harvested on the 14th day to observe the pathological changes (Figure 6C). The morphology of tumor cells in group 1 was almost unaffected. However, after treatment with non-responsive PTX@PPD NPs, the nuclei of some tumor cells were damaged, indicating the apoptosis of tumor cells (Figure 6D). However, after treatment with dual-responsive PTX@PSA NPs, tumor cells were significantly affected, and an extensive range of apoptosis appeared, indicating that dual-responsive PTX@PSA NPs have excellent in vivo tumor inhibition effect (Figure 6E).

Figure $6 \mathbf{F}$ presents the changes in the number of mice surviving after different treatments. Mice treated with saline solution died on day 3; seven of them died on day 21. However, the mice in the dual-responsive PTX@PSA NPs treatment did not die during the treatment and observation period, indicating that the dual-responsive PTX@PSA NPs can prolong the lifespan of mice by inhibiting tumor proliferation.

\section{Long-Term Pathological Study}

The long-term in vivo safety of PSA NPs and PTX@PSA NPs were then further evaluated (Figure 7). Tumor-bearing mice received i.v., injections of $100-\mu \mathrm{l}$ PSA NPs $(0.5 \mathrm{mg} / \mathrm{ml})$ and PTX@PSA NPs $(0.5 \mathrm{mg} / \mathrm{ml})$, respectively. Then, they were sacrificed on the 14th day after treatment, and their five major organs (heart, liver, spleen, lungs, and kidneys) were resected. $\mathrm{H} \& \mathrm{E}$ staining test was performed to evaluate the pathological condition. No apparent pathological changes, such as inflammatory lesions or abnormalities, were observed in the tissue sections of mice.

\section{CONCLUSION}

In conclusion, we reported a dual-responsive nano-carrier constructed from an amphiphilic copolymer composed of -SS- linked PEG and AD. The nano-carrier exhibited dualresponsive characteristics of GSH and acid and could quickly degrade and achieve rapid, controlled release of the drug under the interaction of GSH and acid, which was faster than the response of GSH or acid alone. Therefore, the dual-responsive PTX@PSA NPs possess a good tumor inhibition effect in vivo and in vitro. Furthermore, through the rapid, controlled release of PTX induced by the dual-responsive performance, the inhibitory effect was significantly better than that of non-responsive PTX@ PPD NPs. Based on these conclusions, we believe that the rapid, controlled release of dual-responsive nano-carrier has more potential for clinical applications (Michael and Doherty, 2005).

\section{DATA AVAILABILITY STATEMENT}

The original contributions presented in the study are included in the article/Supplementary Material, further inquiries can be directed to the corresponding authors. 


\section{ETHICS STATEMENT}

The animal study was reviewed and approved by the committee on animals in Nanjing University.

\section{AUTHOR CONTRIBUTIONS}

SL, CP, and YW contributed to conception and design of the study. CS, CQ, and JW organized the database. ZW, XT, and QZ performed the statistical analysis. SL wrote the first draft of the article. CS, CQ, JW, and ZW wrote sections of the article. All

\section{REFERENCES}

Cao, X. T., Vu-Quang, H., Doan, V. D., and Nguyen, V. C. (2021). One-step Approach of Dual-Responsive Prodrug Nanogels via Diels-Alder Reaction for Drug Delivery. Colloid Polym. Sci. 10, 1-9. doi:10.1007/s00396-020-04789-z

Colombo, P., Bettini, R., and Peppas, N. A. (1999). Observation of Swelling Process and Diffusion Front Position during Swelling in Hydroxypropyl Methyl Cellulose (HPMC) Matrices Containing a Soluble Drug. J. Control. Release 61 (1-2), 83-91. doi:10.1016/s0168-3659(99)00104-2

Dai, B., Wu, X., Butch, C. J., Wang, J., Wang, Z. T. S., Wang, Y., et al. (2019). Encapsulating Maytansinoid in $\mathrm{pH}$-Sensitive Nanocarriers: The Importance of Using Extremely Potent Cytotoxic Agents and Fast Release for Nanomedicine to Achieve Tumor Elimination. Nano Res. 12, 1959-1966. doi:10.1007/s12274-019-2464-y

Hou, S.-L., Chen, S.-S., Huang, Z.-J., and Lu, Q.-H. (2019). Dual-responsive Polyphosphazene as a Common Platform for Highly Efficient Drug SelfDelivery. J. Mater. Chem. B 7, 4319-4327. doi:10.1039/c9tb00801b

Hu, Y., Xie, J., Tong, Y. W., and Wang, C.-H. (2007). Effect of PEG Conformation and Particle Size on the Cellular Uptake Efficiency of Nanoparticles with the HepG2 Cells. J. Controlled Release 118 (1), 7-17. doi:10.1016/j.jconrel.2006.11.028

Lammers, T., Kiessling, F., Hennink, W. E., and Storm, G. (2012). Drug Targeting to Tumors: Principles, Pitfalls and (Pre-) Clinical Progress. J. Controlled Release 161 (2), 175-187. doi:10.1016/j.jconrel.2011.09.063

Lappi, A.-K., and Ruddock, L. W. (2011). Reexamination of the Role of Interplay between Glutathione and Protein Disulfide Isomerase. J. Mol. Biol. 409 (2), 238-249. doi:10.1016/j.jmb.2011.03.024

Liu, S., Luo, X., Liu, S., Xu, P., Wang, J., and Hu, Y. (2019). Acetazolamide-Loaded pH-Responsive Nanoparticles Alleviating Tumor Acidosis to Enhance Chemotherapy Effects. Macromol. Biosci. 19 (2), 1800366. doi:10.1002/ mabi.201800366

Luck, A. N., and Mason, A. B. (2013). Structure and Dynamics of Drug Carriers and Their Interaction with Cellular Receptors: Focus on Serum Transferrin. Adv. Drug Deliv. Rev. 65 (8), 1012-1019. doi:10.1016/j.addr.2012.11.001

Maeda, H., Nakamura, H., and Fang, J. (2013). The Epr Effect for Macromolecular Drug Delivery to Solid Tumors: Improvement of Tumor Uptake, Lowering of Systemic Toxicity, and Distinct Tumor Imaging In Vivo. Adv. Drug Deliv. Rev. 65 (1), 71-79. doi:10.1016/j.addr.2012.10.002

Michael, M., and Doherty, M. M. (2005). Tumoral Drug Metabolism: Overview and its Implications for Cancer Therapy. Jco 23 (1), 205-229. doi:10.1200/ jco.2005.02.120

Mura, S., Nicolas, J., and Couvreur, P. (2013). Stimuli-responsive Nanocarriers for Drug Delivery. Nat. Mater 12 (11), 991-1003. doi:10.1038/nmat3776

Petros, R. A., and Desimone, J. M. (2010). Strategies in the Design of Nanoparticles for Therapeutic Applications. Nat. Rev. Drug Discov. 9 (8), 615-627. doi:10.1038/nrd2591

Shi, J., Kantoff, P. W., Wooster, R., and Farokhzad, O. C. (2016). Cancer Nanomedicine: Progress, Challenges and Opportunities. Nat. Rev. Cancer 17, 20-37. doi:10.1038/nrc.2016.108

Sun, Q., Radosz, M., and Shen, Y. (2012). Challenges in Design of Translational Nanocarriers. J. Controlled Release 164 (2), 156-169. doi:10.1016/j.jconrel.2012.05.042

Sun, Q., Zhou, Z., Qiu, N., and Shen, Y. (2017). Rational Design of Cancer Nanomedicine: Nanoproperty Integration and Synchronization. Adv. Mater. 29 (14), 1606628. doi:10.1002/adma.201606628 authors contributed to article revision, read, and approved the submitted version.

\section{FUNDING}

This work was supported by the National Natural Science Foundation of China (Nos. 52103162, 52073111, and 31870952), the Natural Science Foundation of Jiangsu Province (BK20201070), Natural Science Foundation of Jiangsu Higher Education Institution of China (19KJB430013).

Wegiel, B., Gallo, D., Csizmadia, E., Harris, C., Belcher, J., Vercellotti, G. M. et al. (2013). Carbon Monoxide Expedites Metabolic Exhaustion to Inhibit Tumor Growth. Cancer Res. 73 (23), 7009-7021. doi:10.1158/ 0008-5472.can-13-1075

Wu, J. (2021). The Enhanced Permeability and Retention (EPR) Effect: The Significance of the Concept and Methods to Enhance its Application. Jpm 11 (8), 771. doi:10.3390/jpm11080771

Yan, L., Yee, J. A., Boylan, M., and Spallholz, J. E. (1991). Effect of Selenium Compounds and Thiols on Human Mammary Tumor Cells. Biol. Trace Elem. Res. 30 (2), 145-162. doi:10.1007/bf02990350

Yao, K., Gong, G., Fu, Z., Wang, Y., Zhang, L., Li, G., et al. (2020). Synthesis and Evaluation of Cytocompatible Alkyne-Containing Poly( $\beta$-Amino Ester)-Based Hydrogels Functionalized via Click Reaction. ACS Macro Lett. 9 (9), 1391-1397. doi:10.1021/acsmacrolett.0c00545

Ye, M., Han, Y., Tang, J., Piao, Y., Liu, X., Zhou, Z., et al. (2017). A Tumorspecific Cascade Amplification Drug Release Nanoparticle for Overcoming Multidrug Resistance in Cancers. Adv. Mater. 29, 1702342. doi:10.1002/adma.201702342

Yu, H., Chen, J., Liu, S., Lu, Q., He, J., Zhou, Z., et al. (2015). Enzyme Sensitive, Surface Engineered Nanoparticles for Enhanced Delivery of Camptothecin. J. Controlled Release 216, 111-120. doi:10.1016/j.jconrel.2015.08.021

Yu, M., Guo, F., Tan, F., and Li, N. (2015). Dual-targeting Nanocarrier System Based on Thermosensitive Liposomes and Gold Nanorods for Cancer ThermoChemotherapy. J. Controlled Release 215, 91-100. doi:10.1016/j.jconrel.2015.08.003

Zhang, L., Yao, K., Wang, Y., Zhou, Y. L., Fu, Z., Li, G., et al. (2021). Brain-Targeted Dual Site-Selective Functionalized Poly( $\beta$-Amino Esters) Delivery Platform for Nerve Regeneration. Nano Lett. 21 (7), 3007-3015. doi:10.1021/acs.nanolett.1c00175

Zhang, X., Yang, Y., Yao, J., Shao, Z., and Chen, X. (2014). Strong Collagen Hydrogels by Oxidized Dextran Modification. ACS Sustainable Chem. Eng. 2 (5), 1318-1324. doi:10.1021/sc500154t

Zhang, Z., Zhang, X., Ding, Y., Long, P., Guo, J., and Wang, C. (2019). NIRInduced Disintegration of CuS-Loaded Nanogels for Improved Tumor Penetration and Enhanced Anticancer Therapy. Macromol. Biosci. 19 (4), 1800416. doi:10.1002/mabi.201800416

Conflict of Interest: The authors declare that the research was conducted in the absence of any commercial or financial relationships that could be construed as a potential conflict of interest.

Publisher's Note: All claims expressed in this article are solely those of the authors and do not necessarily represent those of their affiliated organizations, or those of the publisher, the editors and the reviewers. Any product that may be evaluated in this article, or claim that may be made by its manufacturer, is not guaranteed or endorsed by the publisher.

Copyright (c) 2021 Liu, Shen, Qian, Wang, Wang, Tang, Zhang, Pan and Ye. This is an open-access article distributed under the terms of the Creative Commons Attribution License (CC BY). The use, distribution or reproduction in other forums is permitted, provided the original author(s) and the copyright owner(s) are credited and that the original publication in this journal is cited, in accordance with accepted academic practice. No use, distribution or reproduction is permitted which does not comply with these terms. 